\title{
MANIPULATING ATTENTION IN COMPUTER GAMES
}

\author{
M. Bernhard, L. Zhang, M. Wimmer \\ Vienna University of Technology \\ Institute of Computer Graphics and Algorithms \\ Favoritenstr. 9-11, Vienna, Austria
}

\begin{abstract}
In computer games, a user's attention is focused on the current task, and task-irrelevant details remain unnoticed. This behavior, known as inattentional blindness, is a main problem for the optimal placement of information or advertisements. We propose a guiding principle based on Wolfe's theory of Guided Search, which predicts the saliency of objects during a visual search task. Assuming that computer games elicit visual search tasks frequently, we applied this model in a "reverse" direction: Given a target item (e.g., advertisement) which should be noticed by the user, we choose a frequently searched game item and modify it so that it shares some perceptual features (e.g., color or orientation) with the target item. A memory experiment with 36 participants showed that in an action video game, advertisements were more noticeable to users when this method is applied.
\end{abstract}

Index Terms - saliency, in-game advertising, inattentional blindness, guided search, attention guidance

\section{INTRODUCTION}

In-game advertising has become an attractive channel for marketing since computer games have emerged as one of the strongest sectors within the entertainment industry, reaching particularly young customer groups. On the other hand, the computer game industry benefits from advertising as a new revenue stream. The big difference to most other entertainment media, e.g., television, is that the user interacts with the computer game, resulting in a highly concentrated, focused, and emotionally fascinating perception of the displayed content.

However, the interactive nature of computer games comes with a severe limitation of the effectiveness of traditional passive forms of advertising, such as product placement on billboards [1]. As computer games frequently require the player to efficiently process many items simultaneously, selective attention creates a perceptual bottleneck where almost exclusively the task-relevant information may pass through.

This work was funded by the FWF-General Purpose Visibility P-21130 project
The amount of attentional resources "left" to process taskirrelevant information, such as advertisements, decreases with increasing difficulty of the task [2]. Since computer games usually impose an intensive task, players are blind for all elements in the game environment which do not contribute taskrelevant information or which are not expected by the user to be related to the task. This effect, named inattentional blindness [3], was demonstrated to be impressively strong, and even conspicuous objects or objects in the foveal field of view may remain unnoticed.

In this paper, we investigate a method to manipulate attention particularly in situations where the task occupies many attentional resources. Attention is manipulated by changing the visual design in a way that takes into account the current task and the resulting attentional behavior. The main idea is to increase the saliency of advertising billboards on a cognitive level. This is achieved by designing task-relevant objects that are frequently searched by the user with colors and other principal perceptual features (e.g. orientation) that are common with the advertising billboards. We establish this method on the theoretical basis of Wolfe's Guided Search [4], a theory explaining top-down guidance in pre-attentive vision and visual search, and present a perceptual study validating the effectiveness.

\section{RELATED WORK}

Directing attention in Virtual Environments (VEs): Though there is a growing academic field concerned with measuring, modeling and predicting visual attention in VEs, there are, to our best knowledge, rather few proposals which attempt to take active control on attention. One approach was proposed by El-Nasr et al. [5], who designed a system which attempts to guide a user's attention in a VE by automatically adjusting lighting conditions, including angles, positions, intensities, and colors of the light sources. A method that operates on the properties of preattentive vision was proposed by Bailey et al. [6]. They used short luminance or warmcool modulations of peripheral image-space regions to direct a user's gaze, which is very sensitive and attracted to sudden changes in the periphery of the fovea. To avoid conscious 
perception of theses modulations, an eye tracker notifies the system when a saccade to the modulated regions is triggered, upon which the modulations have to be stopped. Though this method is very promising for future customer hardware, the requirement of an eye tracker is a severe limitation for many potential applications.

Advertising and information placement in VEs: In-game advertising has emerged as a research area just very recently, and little is known yet about its effectiveness. Research questions which have been addressed mainly concern acceptance, congruity with the schematic context, interactive advertising, effects on players' attitude towards advertised brands, and suitability of game genres for advertising. However, apart from marketing surveys, there are few studies concerned with the perception of advertisements in VEs and games. One factor related to visual attention is schematic congruency of advertisements. Lewis et al. [7] found that advertisements that are schematically incongruent are more conspicuous and hence better remembered. But on the other hand, incongruity may decrease realism significantly (e.g., a soft-drink advertisement in a historical environment). Another factor for the visibility of advertisements is placement. Therefore, Dixit et al. [8] proposed a tool to evaluate the observation densities for surfaces in VEs from recorded viewpoint traces. But the correlation between observation densities and recall of advertisements was rather low. Poor performance in remembering embedded advertisements was also reported by Melzer et al. [9], who conducted a study with a car racing game to evaluate the effect of violence in games on brand memory, and Chaney et al. [1], who carried out a study with a first-person shooter. Our intuition is that embedded advertisements are perceived as parts of the background, which serves as decoration rather than being relevant for the game tasks. Unless they are obviously schematically incongruent or somehow linked with the task, embedded advertisements will remain unnoticed due to the player's inattentional blindness.

\section{BREAKING TROUGH INATTENTIONAL BLINDNESS}

We hypothesize that we can break through inattentional blindness by increasing the saliency of specified objects with respect to top-down factors involved during task execution. Though it is rather difficult to model top-down control for general behavior, there are relatively well examined models for top-down control during a visual search task. Exploiting the fact that in most computer games, visual search tasks are performed frequently (e.g., searching for a health item), we apply an established model in a "reverse" fashion by designing the game in a way that it predicts a high saliency for objects we intend to receive some attention.

Guided Search: According to Treisman and Gelade's The- ory of Feature Integration [10], visual search involves two stages: a parallel preselection and a serial search in preselected regions. The first is denoted as preattentive vision and encodes the visual scene along a number of separable dimensions (e.g. color or orientation) processed in several feature maps. Due to parallel processing, preattentive search is very efficient, but fails to combine features of different channels. Since in many cases, several features have to be combined to distinguish a target from distractors, selective attention is required for feature integration to be carried out serially at the second stage. Overall, preattentive vision optimizes serial search by increasing the saliency of regions bearing important features in order to guide selective attention (e.g. eye movements) towards them. Since the original theory does not explain how top-down control affects search for a particular target, Wolfe proposed Guided Search [4] as an important extension. His model suggests that top-down guidance occurs through a selective manipulation of the impact of particular feature maps. Therefore it is assumed that the visual system holds a representation of a search target where the characteristic features for feature-based search are available. For example, if a yellow line with a leftward slope is the search target, then the feature maps for the yellow channel and the orientation of -45 degrees are weighted higher. Finally the weighted feature maps are combined into one topographical master activation map, and serial search is guided from one position corresponding to peaks of activation to the next until a target is found.

Perceptually adjusted game design: The main idea proposed in this paper is to direct attention intentionally to particular objects by designing the game in a way that those elements become distractors in a visual search task. According to the Guided Search model, we provoke the elements that should be noticed to become "false positives" of serial search since they cause peaks in the master activation map. Now the basic idea is to select an important visual search task and design the corresponding search target in a way that it shares some features with an additional background element that is intended to be recognized. Thus, during the search, the user will find both, the target and the background element.

To this end, we propose the following three requirements to construct an appropriate visual search task: first, the task is performed frequently; second, the position where the target may appear is unpredictable; and third, the target object is linked with positive associations. The first requirement was set to assure that the targeted background item is attended regularly in order to obtain a strong effect. The second property should avoid targets to be found with strategies other than feature-based search, which may occur when a player can learn contextual and spatial constraints for a target's location. The third requirement addresses the emotional impact of our method, due to the fact that advertising is the studied target application. However, the reasons for these choices were 


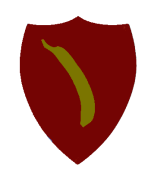

health item 1

( TEST \&

CTRL 1 )
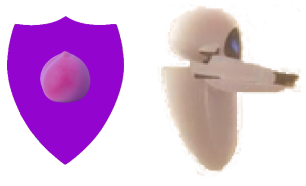

health item 2 ( CTRL 2)

avatar bot

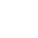

Fig. 1. Elements of the game.

based on intuition, and future studies would be necessary to justify those decisions scientifically.

\section{USER STUDY}

To validate our hypothesis, we created an action video game that contains advertisements of famous brands in the background scene. A memory test was used to measure the effects on users' attention. Compared to eye tracking, this is a more conservative way to observe what has actually been attended, since a user may often look without seeing [11].

We decided to use real brands with high visibility (in the geographical region the study took place) for several reasons: Logos of real and popular brands are a result of a sophisticated design process. They are optimized to be aesthetically pleasant, well recognized and remembered easily. We hence believe that using "home-grown" fictional creations would provide a less realistic application scenario. However, a reasonable concern that can be raised is that using real brands introduces a potential bias due to differences in popularity and saliency of particular designs. Therefore we controlled potential bias in two ways: on the one hand, we used brands of similar popularity (in the region the study took place) according to the BrandZ Ranking Report 2010 [12]. On the other hand, remaining differences are compensated since all interpretations of our results (i.e., statistical tests) are based on comparisons with control conditions where the same advertisements were used and which hence would have been influenced by the same latent factors.

\subsection{The Game}

We authored a representative $2 \mathrm{D}$ action game with a typical game task of popular casual action games and a 3D background where the advertisements were embedded. The game runs in a display window of a resolution of $1,350 \times 700$ pixels and is composed of the following elements (Figure 1):

The avatar (size: $56 \times 76$ pixels) is a grey robot shooting bullets in vertical direction, which can move in all directions (in 2D).

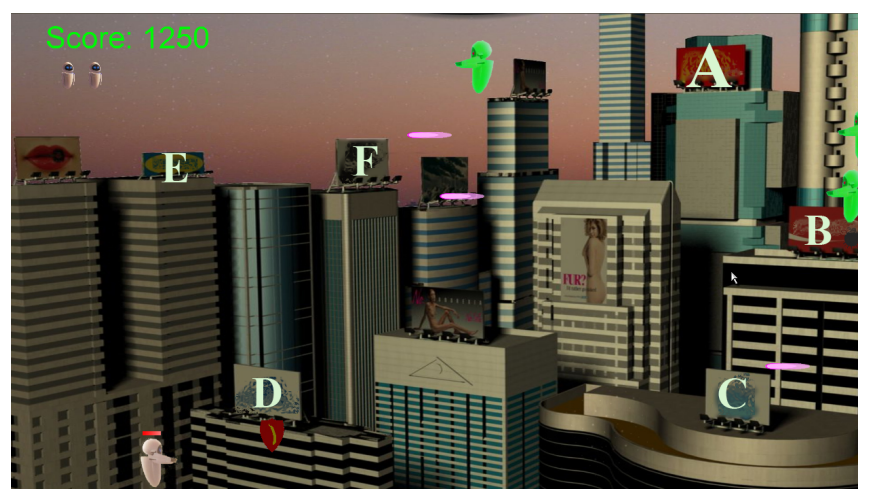

Fig. 2. Screenshot of the action game (TEST condition)

Enemies (size: 60x83) are green robots moving from left to right on a random path, shoot in vertical direction and are killed by one bullet, resulting in an explosion and a gain of 10 game points. A hit by an enemy bullet costs 15 health points.

Flying mines (size: 41x39) have a greyish color, fly on a random path from left to right and cause a reduction of 20 health points when the avatar collides with them.

Health items (size: 80x53) are static, spawned on random positions and collected by moving over with the avatar (+20 health points, +80 game points)

A player starts the game with three lives, each corresponding to 60 health points. The task is to avoid losing all health points (which results in a "Game Over") while trying to gather as many game points as possible. During the game, the frequency of spawning enemies and mines increases constantly. This has the effect that with survival time, difficulty increases, while game points can be earned faster due to an increased frequency of potentially annihilated enemies.

\subsection{Advertisement Placement and 3D Background Scene}

The background was created using 3D Studio Max. It contains a modern city scene with sky scrapers in front of a morning sky. This background is well suited to embed advertisements in a schematically congruent context. Advertisement were placed on six billboards with predefined positions. A screenshot of the game is shown in Figure 2. For the sake of neutrality, we blurred the adverts for this presentation and labeled them with letters A,B,C,D,E and F, which we will use further on as names. Letter A denotes the target advertisement. All brand logos used for the game had only two dominant colors and their momentums ${ }^{1}$ were reasonably high and

\footnotetext{
${ }^{1}$ The momentum of a brand is a measure for its image and popularity and was obtained from the BrandZ Ranking Report 2010 [12]
} 
similar to each other (2 advertisements each with 6,7 and 8, respectively). A belonged to the group with the lowest momentum (6). The positions of the advertisement billboards were selected in a way to obtain a reasonable variation in screen space. To counterbalance potential bias due to position effects, the position of adverts was shifted between participants in a circle (A to position of $\mathrm{B}, \mathrm{B}$ to position of $\mathrm{C}$, and so on) such that in each condition, two (out of twelve) participants saw the same arrangement.

Some artistic freedom was, however, necessary to design a scene with representative aesthetical properties and maximize congruity with the context. Realism and "embeddedness" of the advertisements was increased by adding further advertisement billboards which were not related to any brands or products (e.g., an advertisement against wearing fur) and were not considered in the memory test. The yaw-angle of the billboards textured with advertisements varied with angles between -12 and +14 degrees to the image plane, whereas the pitch-angle was zero for all surfaces. Height and width of the advertising elements varied between $\left(x_{\min }=80, y_{\min }=40\right)$ and $\left(x_{\max }=120, y_{\max }=80\right)$ pixels in the screen-space projection.

The scenes were rendered with a ray tracer (Vray) after the textures of advertising elements were added. The resolution used for rendering was $2,700 \times 1,400$ pixels with a pixel aspect ratio of 1.0, and then rescaled with an image processing tool to fit to the maximum resolution and aspect ratio of the display used in the experiment.

\subsection{Conditions}

We designed three conditions to be tested in this study:

TEST: In this condition, the health item "shield" (Health Item 1 in Figure 1) was designed such that it shared several features with target advertisement A: the shield color and the color, curvature and orientation of the banana drawn on the shield resemble two glyphs in the logo letter of A.

CTRL 1: The first control condition is identical to the TEST condition, apart from using a different health item, a purple shield with a pink blueberry (Health Item 2 in Figure 1). We used this condition to control the self-efficacy of the advertisements and the possible explanation that a potentially higher saliency or popularity of A might have caused the measured effects.

CTRL 2: This condition was similar to the TEST condition, with the difference that A was replaced by another advertisement A' (with same momentum). This second control condition was required to control effects on other advertisements which are due to the differences in the design of the health items, and to control a potential influence of the feature similarity between Health Item 1 and A on the recognition of A in our memory test. Moreover, we can compare the effects of advertising for $\mathrm{A}$ and not advertising for $\mathrm{A}$.

\subsection{Participants}

38 participants $(6$ female) aged between 17 and 38 (mean = 24.3 , stddev $=4.0$ ) volunteered for this study. Each participant was assigned to one condition, which was determined randomly before the participant showed up for the experiment. All except two participants (i.e., 36) were naive about the real purpose of the study, and in case of questions they were told that the study is concerned with reaction times in video games. The two participants who guessed the actual purpose before the experiment were excluded. As the overall procedure can only be performed once per participant (without a significant bias of behavior in a second trial), it was necessary to use a between-subject paradigm, testing each participant in only one of the three conditions. Consequently, each of the three conditions was run with 12 participants. All participants had normal or corrected-to-normal vision. None of them had any type of color blindness.

\subsection{Memory Test}

The memory test participants had to perform was run with a separate application with the following procedure: in each trial the application displayed three advertising elements. One of them had actually been displayed in the game, while the other two were distractors which had not appeared in the game. To also include effects on subconscious memory, we used a forced-choice paradigm, and participants were instructed to guess in case they had difficulties to remember. All in all, a participant had to select 36 times one out of three advertisements. A total of 12 distractor advertisements was used in order to make sure that each advertisement (distracting and in-game advertisement) appeared with the same frequency (6, that is 2 times at each of the 3 locations). The distribution of their momentums was similar to that of in-game advertisements' momentums. Though target advertisement A did not appear in condition CTRL 2 (being replaced by A'), we still tested for recognition of A for participants of this group since we were interested if the similarity between the brand design of $\mathrm{A}$ and the health item has an impact on brand recognition without the actual advertisement being present. To avoid bias due to a difference in the number of trials, we did not include further trials to test whether the replacement, A', was remembered. The combinations were ordered so that repetitions of advertisements in two subsequent trials were avoided and the occurrence frequency of each advertisement was apparently chaotic but still reasonably balanced over time. All participants of the study ran through the same sequence of trials. 

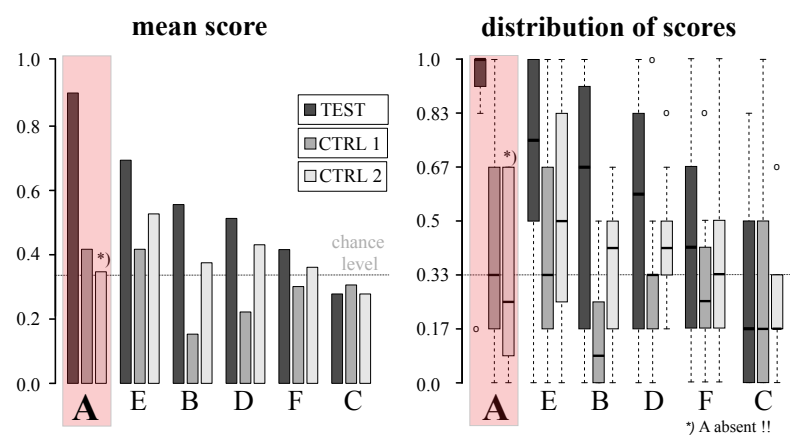

Fig. 3. Results: the average scores are plotted on the left, and the according distribution was visualized with box plots on the right side. The dotted line in the background corresponds to the expected value for random guessing.

\subsection{Procedure}

The game was played on a Sony Vaio VPCEB1S1E notebook running Windows 7, equipped with ATI Mobility graphics hardware and a 15.5" LED TFT display with 16:9 format and $1,366 \times 768$ pixels resolution (a display size that is typical for many casual gaming situations). The experiments were carried out in a sufficiently dimmed room to avoid reflections on the screen using the following protocol after a participant has been introduced to the game:

Playing (3 minutes): The game was timed with a stop watch. In case of a game-over, the watch was paused until the game was restarted.

Distraction (6 minutes): Participants filled a dummy questionnaire and, additionally, were asked some questions orally that required them to access content of their episodic memory (e.g., "What was the first computer game you ever played?"). The purpose was to distract them from the game experience so that the subsequent memory test did not access short-term memory.

Memory test (4 minutes on average): 36 trials (6 per each advertisement in the game)

\section{RESULTS}

We visualized the results in Figure 3, plotting the mean scores and the distributions as box-plots. For statistical analysis (Table 1), we computed an exact Wilcoxon rank sum for pairwise comparisons of the scores between test condition (TEST) and control conditions (CTRL 1 and CTRL 2). Non-parametric tests were indicated due to significant violations of normality and equality of variance assumptions, which are also obvious in the box plots shown in Figure 3. In the following we summarize our main conclusions:

\begin{tabular}{|c|c|c|c|}
\hline \multicolumn{2}{|c|}{ Between conditions (exact Wilcoxon rank sum test, one-tailed, $\mathbf{N}=\mathbf{2 x 1 2}$ ) } \\
\hline Alt. Hypoth. & \multicolumn{3}{|c|}{ Advertisement (1st and 2nd color) } \\
\hline \hline \multirow{3}{*}{ TEST $>$ CTRL 1 } & A (yellow/red) & $\mathrm{E}$ (blue/yellow) & $\mathrm{B}$ (white/red) \\
& $127.5, p=.0003$ & $106.5, p=.02$ & $113.5, p=.007$ \\
\cline { 2 - 4 } & $\mathrm{D}$ (blue/white) & $\mathrm{F}$ (black/white) & $\mathrm{C}$ (blue/white) \\
& $95, p=.096$ & $84.5, p=.24$ & $67.5, p=.62$ \\
\hline \multirow{3}{*}{ TEST $>$ CTRL 2 } & A (yellow/red) & $\mathrm{E}$ (blue/yellow) & $\mathrm{B}$ (white/red) \\
& $136.5, p<0.0001$ & $92.5, p=0.12$ & $91, p=0.14$ \\
\cline { 2 - 4 } & $\mathrm{D}$ (blue/white) & $\mathrm{F}$ (black/white) & $\mathrm{C}$ (blue/white) \\
& $82, p=.30$ & $78, p=.37$ & $63, p=.71$ \\
\hline
\end{tabular}

Table 1. Statistical evaluation of the pairwise differences between conditions for each advertisement. We listed the $\mathrm{W}$-values together with the respective p-values. Denoting colors of the respective advertisements (foreground color/background color) should emphasize potential effects of color similarity (with the yellow/red health item).

Bad memory without manipulating attention: The results for CTRL 1 show that without attentional manipulation, the performance in the memory test was around chance level (33\%) even though participants were exposed 3 minutes to the advertisements. Concerning in particular the results for $\mathrm{A}$, there was almost no difference between CTRL 1 and CTRL 2, even though A was absent in CTRL 2. Testing the hypothesis $H_{1}$ that recognition of $\mathrm{A}$ is better in CTRL 1 compared to CTRL 2 gave an insignificant result (exact Wilcoxon rank sum: $W=81.5, p=0.3$ ). This means that without attentional manipulation, the effectiveness of advertising for $\mathrm{A}$ was barely better than not advertising for A (in a game where a health item sharing abstract features with $\mathrm{A}$ is present).

Highly significant effect for advertisement $\mathrm{A}$ : While the average scores for A in the TEST condition are around $90 \%$, they are around chance level in the control conditions. Regarding the results for the statistical tests (Table 1), we can accept our alternative hypothesis that attention was effectively directed to advertisement A on a highly significant level $(p=.0003)$.

Better memory also for other advertisements than A: Remarkably, also advertisements apart from A were remembered best in TEST. To a certain degree, this can be explained by Guided Search: Advertisement E shared the yellow color and $\mathrm{B}$ the red color with the health item. Hence, scores in TEST and CTRL 2 were better than in CTRL 1, where the health item was neutral. Even more surprising is that the scores of E,B,D and F were also somewhat better in TEST than in CTRL 2, though the same health item was used. The differences are not significant (Table 1), but we suspect a trend in this direction and our guess is that a strong attentional link between a player's tasks and the background also increases the probability that other details get noticed.

No observable effect on gaming performance: We also investigated whether there were any negative effects of the tested method on gaming performance. Therefore we listed the mean of game points and game-overs per condition (Ta- 


\begin{tabular}{|l|c|c|c|}
\hline & TEST & CTRL 1 & CTRL 2 \\
\hline points & $2465 \pm 249$ & $2296 \pm 512$ & $2188 \pm 376$ \\
\hline number of game-overs & $1.27 \pm 0.8$ & $1.17 \pm 0.9$ & $1.25 \pm 1.0$ \\
\hline
\end{tabular}

Table 2. Gaming performance (mean and standard deviation).

ble 2). Surprisingly, the number of game points was highest in the TEST condition, but the effect of condition was not significant (ANOVA: $F(2,33)=1.41, p=0.24^{2}$ ). The number of game overs was marginally lowest in the CTRL 1 condition, but the effect of condition was not significant at all (KruskalWallis: $\chi^{2}=0.1, p=0.95^{3}$ ).

\section{CONCLUSION AND FUTURE WORK}

This study shows that the visual design of task-relevant items, at least of those which are searched frequently, has a significant effect on the recognition of a game's environment. Without feature similarity with task-relevant search targets, elements in the background remain unnoticed (condition CTRL 1 ), at least users are not able to remember them. Whereas, in case of a strong similarity (condition TEST), the respective elements enter a user's memory. Also, with increasing degree of similarity (advertisements E and B in TEST and CTRL 2) to the search target, the performance in the memory test tends to increase. However, though we did not observe a negative effect on task performance in this study, directing attention towards advertisements actually contradicts the goals of a player, who wants to find task-relevant items rather than advertisements. Probably, at some point where this method is applied too intensively, visual distraction may significantly hinder game play. However, this could also be a strategy to volitionally increase the difficulty of a game level, with an increased effectiveness of advertising as side effect.

This work should also be a starting point for the exploration of new avenues for the "reverse" application of visual attention models. For example, the proposed guiding principle would also work to avoid inattentional blindness to other targets than advertisements and find utility for a perceptually optimized game design where unexpected but task-relevant objects are less often overseen by the users.

As this study was preliminary, we maximized the strength of the effect and used 4 features in conjunction (yellow color, red color, orientation and curvature). Since yellow and red are salient colors, the effect of color has probably dominated in this study. However, the clarity of the results should motivate future work to investigate how well the approach works for less salient features and less obvious similarities. Therefore,

\footnotetext{
${ }^{2}$ A Shapiro-Wilk test on residuals and a Levene test showed that normality and equality of variance assumptions were not violated and thus a parametric test could be used.

${ }^{3}$ As the number of game overs was distributed over very few discrete values, normality could not be assumed and a non-parametric test was necessary.
}

one should investigate the effect of particular colors, features and combinations of features by means of a multi-factorial experimental design and analysis. Moreover, the game was (apart from the background) in 2D with a fixed viewpoint, and further studies with games where the viewpoint varies considerably are necessary.

\section{REFERENCES}

[1] I.M. Chaney, K.-H. Lin, and J. Chaney, "The effect of billboards within the gaming environment," J. of Int. Advert., vol. 5, no. 1, pp. 37-45, 2004.

[2] Shawn C. Green and Daphne Bavelier, "Action video game modifies visual selective attention," Nature, vol. 423, no. 6939, pp. 534-7, 2003.

[3] J. Mack and I. Rock, Inattentional Blindness, The MIT Press, Cambridge, MA, 1998.

[4] J.M. Wolfe, "Guided search 2.0: A revised model of visual search," Psychonomic Bulletin and Review, vol. 1, no. 2, pp. 202-238, 1994.

[5] M. S. El-Nasr, T. Vasilakos, C. Rao, and J. Zupko, "Dynamic intelligent lighting for directing visual attention in interactive 3d scenes," IEEE Trans. Comp. Int. and AI in Games, vol. 1, no. 2, pp. 115-134, 2009.

[6] R. Bailey, A. McNamara, N. Sudarsanam, and C. Grimm, "Subtle gaze direction," ACM Trans. Graph., vol. 28, no. 4, pp. 1-14, 2009.

[7] B. Lewis and L. Porter, "In-game advertising effects: Examining player perceptions of advertising schema congruity in a massively multiplayer online role-playing game," J. of Int. Advert., vol. 10, no. 2, pp. 46-60, 2010.

[8] P.N. Dixit and G.M. Youngblood, "Optimal information placement in an interactive 3d environment," in Sandbox '07: Proc. of the 2007 ACM SIGGRAPH Symp. on Video Games, 2007, pp. 141-147.

[9] A. Melzer, B.J. Bushman, and U.G. Hofmann, "When items become victims: Brand memory in violent and nonviolent games," in Entertainment Computing ICEC: 7th Int. Conf. 2008, pp. 325-328, Springer.

[10] A.M. Treisman and G. Gelade, "A feature-integration theory of attention," Cognitive Psychology, vol. 12, no. 1, pp. 97-136, January 1980.

[11] Daniel Memmert, "The effects of eye movements, age, and expertise on inattentional blindness.," Consciousness and Cognition, vol. 15, no. 3, pp. 620-627, 2006.

[12] BrandZ, "2010 full report," www.brandz.com. 